\title{
Endoscopic endonasal transsphenoidal surgery: implementation of an operative and perioperative checklist
}

\author{
Eisha Christian, M.D., ${ }^{1}$ Brianna Harris, M.D., ${ }^{2}$ Bozena Wrobel, M.D., ${ }^{2}$ \\ AND GABRIEl ZADA, M.D. ${ }^{1}$ \\ Departments of ${ }^{I}$ Neurosurgery and ${ }^{2}$ Otolaryngology, Head and Neck Surgery, Keck School of Medicine of \\ University of Southern California, Los Angeles, California
}

\begin{abstract}
Endoscopic endonasal surgery relies heavily on specialized operative instrumentation and optimization of endocrinological and other critical adjunctive intraoperative factors. Several studies and worldwide initiatives have previously established that intraoperative and perioperative surgical checklists can minimize the incidence of and prevent adverse events. The aim of this article was to outline some of the most common considerations in the perioperative and intraoperative preparation for endoscopic endonasal transsphenoidal surgery. The authors implemented and prospectively evaluated a customized checklist at their institution in 25 endoscopic endonasal operations for a variety of sellar and skull base pathological entities. Although no major errors were detected, near misses pertaining primarily to missing components of surgical equipment or instruments were identified in 9 cases (36\%). The considerations in the checklist provided in this article can serve as a basic template for further customization by centers performing endoscopic endonasal surgery, where their application may reduce the incidence of adverse or preventable errors associated with surgical treatment of sellar and skull base lesions. (http://thejns.org/doi/abs/10.3171/2014.7.FOCUS14360)
\end{abstract}

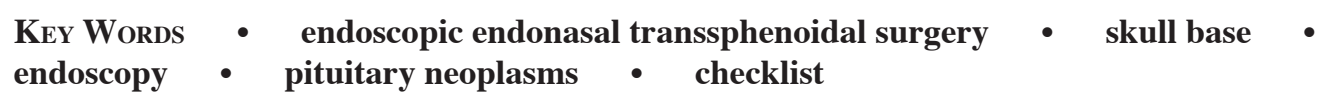

$\mathrm{S}$ EVERAL studies and worldwide initiatives have previously established that intraoperative and perioperative surgical checklists can minimize the incidence of and prevent adverse events..$^{5,9,15,16}$ Checklists for surgical procedures have been used on an international basis, and major reductions in morbidity and complication rates have been achieved. ${ }^{6,12}$ Endoscopic endonasal surgery is highly dependent on the technology and instrumentation that are integral to successful completion of the operation; the unavailability or dysfunction of any of these components may prevent the operation from being completed. ${ }^{4}$ Although surgical practices in endonasal endoscopic surgery may vary greatly from one institution to another, we aimed to develop and pilot a working model for an intraoperative and perioperative checklist for the surgical treatment of pituitary tumors and other parasellar lesions. As endoscopic transsphenoidal surgery becomes more commonplace, and with its increasing complexity with respect to imaging, instrumentation, and endocri-

\footnotetext{
Abbreviations used in this paper: $\mathrm{ACTH}=$ adrenocorticotropic hormone; $\mathrm{BMP}=$ basic metabolic panel; $\mathrm{CBC}=$ complete blood count; $\mathrm{CD}=$ Cushing's disease; $\mathrm{DDAVP}=$ desmopressin; $\mathrm{DI}=$ diabetes insipidus; FSH = follicle-stimulating hormone; $\mathrm{GH}=$ growth hormone; IGF-I = insulin-like growth factor-I; LH = luteinizing hormone; $\mathrm{PRL}=$ prolactin; $\mathrm{PT}=$ prothrombin time; $\mathrm{PTT}=$ partial thromboplastin time; $\mathrm{TSH}=$ thyroid-stimulating hormone; $\mathrm{T} 4=$ thyroxine.
}

nological testing, it will probably become increasingly important to standardize this procedure by implementing an intraoperative checklist. The aims of this article were to outline the necessary considerations in the perioperative and intraoperative period prior to performing an endoscopic endonasal approach to the sellar region and to describe our early experience with implementation of this surgical checklist.

\section{Methods}

A 3-part surgical checklist was devised, consisting of preoperative, intraoperative, and immediate postoperative sections. Data points for each section (summarized in Tables 1 and 2) were entered into a Web-based questionnaire completed prior to and at the conclusion of endoscopic endonasal skull base cases between August 2013 and June 2014. The online questionnaire was stored on a REDCAP database with password protection. All cases were treated by the same neurosurgical attending physician; an otolaryngologist was present for the extended approaches and for those requiring pedicled nasoseptal flap reconstruction..$^{10}$ An extended timeout was conducted as part of the routine institutional surgical timeout. In addition, a closeout portion was filled out at the end of each operation. All missing data, near misses, and perioperative errors were recorded and analyzed. 


\section{E. Christian et al.}

The checklist is described in detail below to discuss the rationale behind each data point for each of the $3 \mathrm{sec}-$ tions (preoperative, intraoperative, and immediate postoperative).

\section{Preoperative Checklist Considerations (Table 1)}

History and Physical Examination. Prior to any consideration for surgical intervention for pituitary adenomas or other sellar lesions, a focused patient history and detailed physical examination is required. In many cases, close collaboration with an endocrine specialist has already been established. Detailed questions should always be asked to screen for Cushing's disease (CD), acromegaly, fatigue, amenorrhea/galactorrhea, diminished libido, headache, visual loss, and other common symptoms and conditions associated with pituitary lesions. The preoperative workup should also include ophthalmology, otolaryngology, and endocrinology consultations as needed. Any patients harboring lesions in proximity to the optic apparatus should undergo formal visual testing, including visual field examination, by a neuroophthalmologist.

Laboratory Tests. Once a thorough history and physical examination is completed, a routine preoperative laboratory panel that includes complete blood count (CBC), basic metabolic panel (BMP), prothrombin time (PT), partial thromboplastin time (PTT), and a type and screen should be obtained. In addition, an endocrine panel including prolactin (PRL), fasting morning cortisol, adrenocorticotropic hormone (ACTH), thyroid-stimulating hormone (TSH), free thyroxine (T4), growth hormone $(\mathrm{GH})$, insulin-like growth factor-I (IGF-I), luteinizing hormone (LH), follicle-stimulating hormone (FSH) (in women), and free testosterone level (in men) should be part of the preoperative workup to identify any underlying hormonal abnormality. One major consideration is to always ensure that a PRL level has been checked to avoid surgically treating a tumor that may be treatable with medications. A serum PRL level $>200 \mu \mathrm{g} / \mathrm{L}$ is typically diagnostic of a prolactinoma-these can be treated medically and will need to be identified prior to any surgical decision making. A serum PRL $\leq 200 \mu \mathrm{g} / \mathrm{L}$ in a patient with a macroadenoma points to pituitary stalk effect (decreased delivery of dopamine) rather than tumor secretion of PRL. Furthermore, one must always consider the potential for a phenomenon known as the hook effect, which may occur in macroprolactinomas with extremely high levels of circulating PRL that may overwhelm the immunoassay and result in an artificially low or even normal PRL level. Dilution of serum samples must be specifically requested if suspicion of the hook effect is high enough. ${ }^{8,13,14}$

Of the major anterior pituitary hormonal axes, the two that typically require the most attention with regard to hormonal replacement prior to any intervention are the cortisol and thyroid axes. Medical replacement of hypofunctioning cortisol or thyroid axes should be implemented prior to any operation, typically in collaboration with an endocrinologist. A fasting morning cortisol test should be included in the preoperative workup and determines the need for stress-dose steroids in the operating room. Ideally, these levels should be measured between 8:00 and 9:00 a.m., when basal cortisol levels are at a peak. Appropriate stimulation testing, such as a cosyntrophin stimulation test, should be performed on an as-needed basis prior to any operation. ${ }^{8,13,14}$ Free T4 and TSH levels should also be checked prior to any intervention and treated according to the findings.

Imaging. Imaging should include an MRI study of the brain and sellar region with and without contrast. For the

TABLE 1: Preoperative checklist for endoscopic endonasal surgery*

\begin{tabular}{|c|c|c|}
\hline Category & Item & Subitem \\
\hline \multicolumn{3}{|c|}{ History \& Physical Exam } \\
\hline \multirow[t]{4}{*}{ Laboratory Tests } & general labs & CBC, BMP, PT/PTT, INR, type \& screen \\
\hline & hormonal labs & $\begin{array}{l}\text { PRL, a.m. cortisol, ACTH, TSH, free T4, FSH, LH, } \\
\text { free testosterone, GH, IGF-I }\end{array}$ \\
\hline & stimulation testing (as needed) & \\
\hline & PRL level dilutions (as needed to rule out hook effect) & \\
\hline \multirow[t]{4}{*}{ Imaging } & MRI of sella w/ or w/o contrast; sagittal \& coronal sequences & \\
\hline & $\mathrm{CT}$ (as needed) & \\
\hline & MRA or CTA (as needed to rule out vascular lesion) & \\
\hline & MRI or CT for neuronavigation & \\
\hline \multirow[t]{2}{*}{ Informed Consent } & procedure & fat/fascial graft, lumbar drain \\
\hline & tumor bank/clinical trial & \\
\hline Meds & $\begin{array}{l}\text { corticosteroid, thyroid replacement therapy } \\
\text { discontinue blood thinners }\end{array}$ & hydrocortisone, levothyroxine \\
\hline \multirow[t]{3}{*}{ Consultations } & endocrinology & \\
\hline & neuroophthalmology (visual field testing) & \\
\hline & medical clearance & \\
\hline
\end{tabular}

* CTA = CT angiography; INR = international normalized ratio; Meds = medications; MRA = MR angiography. 
majority of sellar-based lesions, sagittal and coronal MRI sequences are preferred. A CT scan of the head obtained without contrast can also be helpful to detect intracranial calcification (i.e., with craniopharyngiomas) and is the preferred modality for patients who are unable to undergo MRI studies. A CT scan is also helpful for detailing the bony sphenoid sinus anatomy, including sphenoid septa, although an MRI study is also typically adequate for these purposes. ${ }^{17}$ Close attention should also be paid to the anatomy of the paranasal sinuses and configuration of any sphenoid sinus septations, degree of pneumatization of the sphenoid sinus, and morphology of the sellar floor. Furthermore, the course of the internal carotid arteries and intercarotid distance should always be considered prior to planning any endonasal endoscopic skull base approach. Of course, any prior surgical history and methods for reconstruction must always play a major role in consideration of one surgical approach over another. Based on the planned operation, anatomy of the sellar floor and paranasal sinuses, and prior surgical history, the surgeon should plan according to whether intraoperative neuronavigation will be used, and this imaging should be performed ahead of time. We routinely use intraoperative neuronavigation in direct sellar approaches where a "flat" sellar floor is identified, in most extended approaches, and in all repeat operations. ${ }^{17}$

Informed Consent. As with any neurosurgical procedure, a candid discussion regarding the goals, risks, and benefits of as well as alternatives to surgery must take place with the patient as part of the informed consent process. The consent should address additional measures such as an abdominal or thigh tissue graft or lumbar drain, as needed. Additional consent forms for the purpose of tumor analysis (i.e., genomic or molecular studies) and storage, or other clinical research protocols, should also be obtained prior to starting the intervention.

\section{Intraoperative Checklist (Table 2)}

Immediately prior to any endonasal endoscopic operation for pituitary lesions, consideration of some or all of the following factors may reduce or even prevent the development of intraoperative complications, align the workflow goals of the surgical and operating room team, and ensure that all required equipment is available and functional before commencing the procedure.

Surgical Timeout and Medications. As with most surgical checklists, initial confirmation of the patient, procedure, and operative site are critical, in addition to a review of patient allergies and medications to be administered prior to and during the operation. Close communication with the anesthesiology team is imperative to assure that the patient receives the correct doses of medications required. Perioperative antibiotics (most commonly cefazolin) should be administered. Patients with evidence of hypocortisolemia should receive cortisol replacement (hydrocortisone or dexamethasone) during surgery, whereas at our institution, patients with normal preoperative cortisol levels who are undergoing selective adenomectomy do not undergo routine cortisol replacement. For patients with $\mathrm{CD}$ who are undergoing resec- tion of a pituitary adenoma, the checklist must include a reminder that no corticosteroids are to be administered.

Imaging. The surgical team should ensure that the desired imaging is available and easily accessible in the operating room prior to starting the operation. For a majority of sellar-based lesions, sagittal and coronal MRI studies are preferred. Close attention should again be paid to the anatomy of the paranasal sinuses and configuration of any sphenoid sinus septations, degree of pneumatization of the sphenoid sinus, and morphology of the sellar floor. Furthermore, the course of the internal carotid arteries and intercarotid distance should be reviewed prior to surgery. The degree of tumor invasion into surrounding structures, proximity of critical blood vessels, and location of the normal pituitary gland and stalk relative to the lesion should be reviewed. If intraoperative neuronavigation is to be used, the team should ensure that the correct image sequences and equipment is available.

Adjunctive Measures. Once the patient is intubated, the endotracheal tube should be mobilized to the left side of the mouth. An arterial line is preferred for blood pressure monitoring. An orogastric tube is inserted, which will be used once surgery is completed to suction any surgical fluid that was swallowed. Another alternative is to pack the oropharynx with moist throat packs or gauze. If throat packs are used, they will need to be included in the surgical count at the beginning and end of the operation. Additional adjunctive measures that require planning and discussion with the anesthesiology and nursing teams include insertion of a Foley catheter, which we use only for extended procedures, and plans for inserting a lumbar drain. If a lumbar drain is inserted prior to the operation, clear communication with the anesthesia team must take place to assure that the drain settings are optimized.

Positioning. The patient is placed in the supine position with the head mildly flexed and rotated approximately $10^{\circ}$ toward the surgeon, with the head elevated above the thorax to optimize venous outflow. Intraoperative neuronavigation can then be registered to verify accuracy. At this point, the nose is packed with lidocaine-soaked cottonoids or a nasal decongestant such as oxymetazoline. The nose is then prepared with povidone-iodine or another cleansing agent after the cottonoids are removed. The abdomen (or lateral thigh) should also be prepared and draped for a potential fat and/or fascia graft. Any plans for a pedicled nasoseptal flap should be discussed prior to starting the operation.

Equipment. Prior to any operation, it is mandatory that the surgeon and operating team confirm that the surgical instruments and equipment necessary to perform the procedure are available and sterile. Endoscopic skull base surgery requires that each component of the visualization system, which includes the telescope, camera, light source, fiberoptic cable, and display monitor, functions in an ideal fashion. If any of these components are not working properly, the ability to perform the operation may be compromised. This has been referred to as a "clock-gear" mechanism, in which the entire endoscopy setup relies heavily on each individual component. ${ }^{2}$ In addition to the 
TABLE 2: Intraoperative checklist for endoscopic endonasal surgery*

\begin{tabular}{|c|c|c|}
\hline Category & Item & Subitem \\
\hline Surgical Timeout & patient ID, procedure, allergies, meds & \\
\hline \multirow[t]{2}{*}{ Meds } & antibiotics & Ancef, vancomycin \\
\hline & corticosteroids & hydrocortisone, dexamethasone \\
\hline Imaging & $\begin{array}{l}\text { MRI of sella w/ or w/o contrast; sagittal \& coronal sequences } \\
\text { displayed in OR }\end{array}$ & \\
\hline \multirow[t]{5}{*}{ Adjunctive Measures } & endotracheal tube positioning & routine \\
\hline & radial arterial line & routine \\
\hline & orogastric tube or oral packing & routine \\
\hline & lumbar drain & extended approaches \\
\hline & Foley catheter & extended approaches \\
\hline \multirow[t]{2}{*}{ Positioning } & head elevated above thorax & \\
\hline & flexion/extension for extended approaches & \\
\hline Neuronavigation & registration performed w/ good accuracy & \\
\hline \multirow[t]{3}{*}{ Preparation } & nasal & Afrin, lidocaine w/ epinephrine, iodine prep \\
\hline & abdomen or thigh for fat/fascial harvest & \\
\hline & pedicled nasoseptal flap & extended approaches \\
\hline \multirow[t]{12}{*}{ Equipment } & endoscopy system & $0^{\circ}, 30^{\circ}, \& 45^{\circ}$ telescopes \\
\hline & & camera \\
\hline & & light source \& fiberoptic cable \\
\hline & & HD monitor \\
\hline & & Clearvision or other lens irrigator \\
\hline & & recording equipment \\
\hline & microscope & as needed for backup \\
\hline & sinus debrider & \\
\hline & sinus \& skull base instruments & \\
\hline & micro-Doppler & \\
\hline & tumor resection instruments & Nico Myriad, CUSA, SonoPet \\
\hline & reconstruction materials & DuraSeal, fibrin glue, Porex plate \\
\hline \multirow[t]{2}{*}{ Surgical Terminus } & removal of throat packs or OGTT suctioning & \\
\hline & pupillary/visual check (prior to leaving OR) & \\
\hline
\end{tabular}

* CUSA = Cavitron ultrasonic surgical aspirator; $\mathrm{HD}=$ high definition; OGTT = oral glucose tolerance test; OR = operating room; prep = preparation.

endoscopy system, the surgeon should ensure that the correct skull base and transsphenoidal instruments are available, in addition to the sinus debrider, high-speed drill, lens irrigating systems, micro-Doppler instrument for carotid localization, ${ }^{7}$ and/or additional mechanical tumor resection devices such as the ultrasonic aspirator.

Surgical Terminus. Once surgery is completed and a sling dressing is in place, the throat packs must be removed. If an oral glucose tolerance test was used, the anesthesiologist will suction out gastric contents prior to extubation. Immediately following extubation and prior to transfer to the recovery room, visual status is always verified to ensure that no acute compression of the optic apparatus has occurred secondary to an expanding hematoma or graft insertion.

\section{Postoperative Checklist Considerations (Table 3)}

Postoperative Examination and Orders. Postoperative care begins with an immediate neurological exami- nation with attention to visual acuity and fields. Postoperative orders should include screening parameters for diabetes insipidus (DI), which include checking sodium levels every 6-12 hours. In addition, the nursing staff should be instructed to send an immediate urine sample for specific gravity and sodium tests for urine output $>$ $250 \mathrm{ml} /$ hour for 2 consecutive hours. If a patient has low preoperative cortisol axis function, high-dose hydrocortisone should be tapered down to a physiological dose over the next week or so.

Postoperative Day 1 laboratory tests should include close monitoring for new hypocortisolemia in patients with normal preoperative hypothalamic-pituitary-adrenal axis function who were not given intraoperative corticosteroids; this is often done by obtaining daily morning fasting cortisol levels and initiating cortisol replacement for patients with low values and/or symptoms consistent with hypocortisolemia. In patients with acromegaly, a fasting morning $\mathrm{GH}$ level should be obtained on postop- 


\section{Checklist for endoscopic transsphenoidal surgery}

TABLE 3: Postoperative checklist for endoscopic endonasal surgery*

\begin{tabular}{|c|c|c|}
\hline Category & Item & Subitem \\
\hline Physical Exam & $\begin{array}{l}\text { general neurological exam } \\
\text { visual acuity \& fields }\end{array}$ & \\
\hline Laboratory Tests Ordered & $\begin{array}{l}\text { sodium levels } \\
\text { monitoring for DI }\end{array}$ & $\begin{array}{l}\text { every } 6 \text { or } 12 \mathrm{hrs} \\
\text { if UOP }>250 \mathrm{ml} / \mathrm{hr} \text { for } 2 \text { consecutive hrs, send } \\
\text { immediate specific gravity \& sodium }\end{array}$ \\
\hline & next-day a.m. labs & $\begin{array}{l}\text { CD: cortisol level every } 6 \text { hrs } \\
\text { acromegaly: a.m. GH level } \\
\text { HPA axis monitoring: cortisol level every a.m. } \\
\text { prolactinoma: PRL level every a.m. }\end{array}$ \\
\hline $\begin{array}{l}\text { Imaging } \\
\text { Special Orders }\end{array}$ & $\begin{array}{l}\text { MRI of sella w/ or w/o contrast; sagittal \& coronal sequences } \\
\text { lumbar drain specifications } \\
\text { set time for removal of nasal packing (if used) }\end{array}$ & either w/in 72 hrs or in 3 mos \\
\hline Meds & $\begin{array}{l}\text { antibiotics } \\
\text { hormone replacement } \\
\text { DDAVP }\end{array}$ & \\
\hline Discharge Planning & $\begin{array}{l}\text { sodium level on postop Day } 7 \\
\text { follow-up w/ endocrinologist \& ophthalmologist }\end{array}$ & \\
\hline
\end{tabular}

* HPA = hypothalamic-pituitary-adrenal; UOP = urine output.

erative Days 1 and 2; levels $<2 \mathrm{ng} / \mathrm{ml}$ typically predict long-term remission, whereas higher levels are associated with higher rates of recurrence. ${ }^{11}$ Additional laboratory tests include cortisol levels every 6 hours in patients with CD and daily morning PRL levels for patients with prolactinomas. ${ }^{1,3}$ If early DI develops, we prefer to use subcutaneous desmopressin (DDAVP) initially, and then transition to oral or nasal spray DDAVP as needed, in anticipation of discharge planning.

Special Orders. Although nasal packing is not routinely used, additional instructions include removal of nasal packing at any specified postoperative time point when used and specific lumbar drain management specifications. For standard pituitary adenomas, we typically obtain an MRI study of the sella 3 months after surgery. However, for nonadenomatous lesions or extended approaches we often obtain postoperative imaging within 72 hours of surgery. A repeat sodium level, obtained on an outpatient basis, should be checked on or around postoperative Day 7 to evaluate for delayed hyponatremia. ${ }^{18}$ Discharge planning should include follow-up with the neurosurgeon, endocrinologist, and ophthalmologist.

\section{Results}

We surveyed a total of 25 patients (Table 4) with 16 endoscopic endonasal approaches for pituitary adenomas, 3 for Rathke cleft cysts, 1 arachnoid cyst, and 1 case of lymphocytic hypophysitis. The checklist was also applied to additional anterior skull base endoscopic cases, including 3 anterior fossa CSF leak reconstructions and 1 clival chordoma. Twenty-three cases were direct endoscopic transsphenoidal approaches and two were extended endonasal cases. We used nasoseptal flap reconstruction in 7 cases.
The checklist was readily adopted by nursing and anesthesia colleagues without any barriers to implementation.

Our preoperative checklist survey (Table 5) had no missed or incomplete sections. A majority of checkpoints in this section are part of our institutional preoperative checklist and therefore were redundant checkpoints. It is important to note that thyroid replacement and steroid therapy are not part of our institutional list and therefore might be missed or overlooked if not thoroughly reviewed before an operation.

During implementation of the intraoperative checklist (Table 6), no major surgical errors were identified.

\section{TABLE 4: Patient clinical data}

\begin{tabular}{lc}
\multicolumn{1}{c}{ Finding \& Procedure } & No. of Patients \\
\hline total & 25 \\
diagnosis & \\
pituitary adenoma & 16 \\
Rathke cleft cyst & 3 \\
arachnoid cyst & 1 \\
lymphocytic hypophysitis & 1 \\
CSF leak repair & 3 \\
chordoma & 1 \\
procedure & \\
standard endoscopic transsphenoidal & 23 \\
extended approach & 2 \\
lumbar drain (inserted prior to procedure) & 1 \\
abdominal fat graft (site prepped) & 14 \\
thigh graft (site prepped) & 11 \\
nasoseptal flap & 7 \\
\hline
\end{tabular}




\section{E. Christian et al.}

TABLE 5: Preoperative checklist results*

\begin{tabular}{lc}
\hline \multicolumn{1}{c}{ Checklist Item } & No. of Patients \\
\hline preop workup & \\
history \& physical & 25 \\
basic labs (CBC, BMP) & 25 \\
coagulation studies (PTT, INR) & 25 \\
chest radiograph & 25 \\
EKG & 25 \\
pregnancy test & 12 \\
blood type \& screen & 25 \\
blood available & 8 \\
PRL & 17 \\
a.m. cortisol & 18 \\
thyroid studies & 18 \\
IGF-I & 15 \\
consent & 25 \\
tumor bank consent & 14 \\
meds & \\
blood thinners discontinued & 7 \\
thyroid replacement therapy & 3 \\
intraop steroids & 6 \\
antibiotics & 25 \\
\hline \multirow{2}{*}{ EKG = electrocardiogram. } &
\end{tabular}

However, 9 missing surgical components (near misses) that could have delayed or impeded progress during surgery were identified. These items (Table 7) included missing neuronavigation setup/equipment, Clearvision or other lens irrigator, micro-Doppler, recording equipment, and selected endoscopic skull base instruments.

\section{Discussion}

We implemented a standardized and customized perioperative checklist designed for endoscopic pituitary and skull base surgery. The checklist was successfully used in 25 nonconsecutive cases over a 9-month period as a pilot analysis. We aimed to share our early experience and the specifics of our checklist as we move toward routine implementation of the checklist in all endoscopic endonasal cases. In our experience, the preoperative checklist proved to be redundant with the institutional checklist except for the endocrinological workup. The checklist was most useful in ensuring that all necessary equipment was available and functional prior to commencement of the operation. Although no major errors were detected (e.g., failure to detect a prolactinoma prior to transsphenoidal pituitary operation), the checklist was valuable in identifying missing key components of the operation in 9 cases (36\% of operations). This checklist is especially helpful for new operating room personnel or in institutions that are just beginning to use endoscopy.

The limitations of this pilot prospective study are its implementation in a single center by a single surgeon in a
TABLE 6: Intraoperative checklist*

\begin{tabular}{|c|c|}
\hline Checklist Item & No. of Patients \\
\hline \multicolumn{2}{|l|}{ surgical start } \\
\hline timeout & 25 \\
\hline neuronavigation available & 22 \\
\hline MRI available & 22 \\
\hline CT & 3 \\
\hline CTA/MRA & 1 \\
\hline \multicolumn{2}{|l|}{ surgical positioning \& adjuncts } \\
\hline endotracheal tube positioned/taped & 25 \\
\hline Mayfield pins & 18 \\
\hline head above thorax & 25 \\
\hline leg squeezers/SCDs & 25 \\
\hline radial artery line & 25 \\
\hline Foley catheter & 10 \\
\hline lumbar drain & 3 \\
\hline OGTT & 2 \\
\hline throat packs & 23 \\
\hline \multicolumn{2}{|l|}{ surgical prep } \\
\hline Afrin in nasal cavity & 25 \\
\hline Betadine prep & 25 \\
\hline abdomen prepped & 14 \\
\hline thigh prepped & 11 \\
\hline \multicolumn{2}{|l|}{ endoscopy equipment } \\
\hline $0^{\circ}, 30^{\circ}, \& 45^{\circ}$ scopes & 25 \\
\hline camera & 25 \\
\hline light source/fiberoptic cable & 25 \\
\hline HD monitor & 25 \\
\hline Clearvision lens irrigation & 23 \\
\hline recording & 24 \\
\hline \multicolumn{2}{|l|}{ surgical equipment } \\
\hline sinus debrider & 25 \\
\hline skull base instruments & 24 \\
\hline micro-Doppler & 23 \\
\hline tumor resection: Nico Myriad & 2 \\
\hline FloSeal/hemostatic agent & 6 \\
\hline reconstruction materials: DuraSeal/Duragen & 3 \\
\hline \multicolumn{2}{|l|}{ surgical terminus } \\
\hline throat packs removed & 23 \\
\hline vision checked & 25 \\
\hline counts correct & 25 \\
\hline
\end{tabular}

* $\mathrm{SCD}=$ sequential compression device.

nonconsecutive pattern. More widespread and consistent analysis following routine use of this checklist via a multiinstitutional format will provide more valid information pertaining to the benefits of its adoption. Nevertheless, we believe that reporting the early results of this pilot analysis and sharing our experience with the customized checklist may facilitate this process and lead to more rapid adoption of similar checklists worldwide. 
TABLE 7: Incomplete or missing components constituting "near misses"

\begin{tabular}{lc}
\hline \multicolumn{1}{c}{ Component } & No. of Cases \\
\hline recording equipment & 1 \\
neuronavigation & 3 \\
Clearvision lens irrigation & 2 \\
micro-Doppler & 2 \\
skull base tray & 1 \\
\hline
\end{tabular}

\section{Conclusions}

A successful approach to pathological entities in the sellar region begins in the preoperative period with a thorough workup and attention to the patient's endocrinological and visual function. Intraoperatively, ensuring availability and functionality of the right equipment, positioning, and any adjunctive measures is likely to optimize workflow and improve patient outcomes. The considerations in the checklist provided in this review can serve as a basic tool for further customization by individuals performing endoscopic transsphenoidal or anterior skull base surgery, and the use of such tools may reduce the incidence of adverse or preventable errors associated with surgical treatment of sellar and skull base lesions.

\section{Disclosure}

The authors report no conflict of interest concerning the materials or methods used in this study or the findings specified in this paper.

Author contributions to the study and manuscript preparation include the following. Conception and design: Christian, Wrobel, Zada. Acquisition of data: Christian, Wrobel, Zada. Analysis and interpretation of data: Christian, Zada. Drafting the article: Christian, Harris, Zada. Critically revising the article: Christian, Zada. Reviewed submitted version of manuscript: Christian, Zada. Approved the final version of the manuscript on behalf of all authors: Christian. Study supervision: Zada.

\section{References}

1. Amar AP, Couldwell WT, Chen JC, Weiss MH: Predictive value of serum prolactin levels measured immediately after transsphenoidal surgery. J Neurosurg 97:307-314, 2002

2. Cappabianca P, Cavallo LM, Esposito F, de Divitiis E: Endoscopic endonasal transsphenoidal surgery: procedure, endoscopic equipment and instrumentation. Childs Nerv Syst 20:796-801, 2004

3. Chen JC, Amar AP, Choi S, Singer P, Couldwell WT, Weiss MH: Transsphenoidal microsurgical treatment of Cushing disease: postoperative assessment of surgical efficacy by application of an overnight low-dose dexamethasone suppression test. J Neurosurg 98:967-973, 2003

4. Comer BT, Young AB, Gal TJ: Impact of endoscopic surgical techniques on efficiency in pituitary surgery. Otolaryngol Head Neck Surg 145:732-736, 2011
5. Conley DM, Singer SJ, Edmondson L, Berry WR, Gawande AA: Effective surgical safety checklist implementation. J Am Coll Surg 212:873-879, 2011

6. Da Silva-Freitas R, Martín-Laez R, Madrazo-Leal CB, Villena-Martin M, Valduvieco-Juaristi I, Martínez-Agüeros JA, et al: [Establishment of a modified surgical safety checklist for the neurosurgical patient: Initial experience in 400 cases.] Neurocirugia (Astur) 23:60-69, 2012 (Span)

7. Dusick JR, Esposito F, Malkasian D, Kelly DF: Avoidance of carotid artery injuries in transsphenoidal surgery with the Doppler probe and micro-hook blades. Neurosurgery 60 (4 Suppl 2):322-329, 2007

8. Guinto G, Cohn F, Perez-de la Torre R, Gallardo M: Pituitary macroadenomas: transsphenoidal approach, in Sekhar LN, Fessler RG (eds): Atlas of Neurosurgical Techniques: Brain. New York: Thieme Medical, 2006, pp 670-679

9. Haynes AB, Weiser TG, Berry WR, Lipsitz SR, Breizat AH, Dellinger EP, et al: A surgical safety checklist to reduce morbidity and mortality in a global population. N Engl J Med 360:491-499, 2009

10. Kassam AB, Gardner P, Snyderman C, Mintz A, Carrau R: Expanded endonasal approach: fully endoscopic, completely transnasal approach to the middle third of the clivus, petrous bone, middle cranial fossa, and infratemporal fossa. Neurosurg Focus 19(1):E6, 2005

11. Krieger MD, Couldwell WT, Weiss MH: Assessment of longterm remission of acromegaly following surgery. J Neurosurg 98:719-724, 2003

12. Lyons MK: Eight-year experience with a neurosurgical checklist. Am J Med Qual 25:285-288, 2010

13. Parent A, Flotte E: Pediatric pituitary adenomas, in Albright AL, Pollack I, Adelson D (eds): Principles and Practice of Pediatric Neurosurgery, ed 2. New York: Thieme, 2008, pp 579-584

14. Pereira O, Bevan JS: Preoperative assessment for pituitary surgery. Pituitary 11:347-351, 2008

15. Semel ME, Resch S, Haynes AB, Funk LM, Bader A, Berry WR, et al: Adopting a surgical safety checklist could save money and improve the quality of care in U.S. hospitals. Health Aff (Millwood) 29:1593-1599, 2010

16. Weiser TG, Haynes AB, Lashoher A, Dziekan G, Boorman DJ, Berry WR, et al: Perspectives in quality: designing the WHO Surgical Safety Checklist. Int J Qual Health Care 22:365-370, 2010

17. Zada G, Agarwalla PK, Mukundan S Jr, Dunn I, Golby AJ, Laws ER Jr: The neurosurgical anatomy of the sphenoid sinus and sellar floor in endoscopic transsphenoidal surgery. Clinical article. J Neurosurg 114:1319-1330, 2011

18. Zada G, Liu CY, Fishback D, Singer PA, Weiss MH: Recognition and management of delayed hyponatremia following transsphenoidal pituitary surgery. J Neurosurg 106:66-71, 2007

Manuscript submitted June 16, 2014.

Accepted July 11, 2014.

Please include this information when citing this paper: DOI: 10.3171/2014.7.FOCUS14360.

Address correspondence to: Eisha Christian, M.D., 1200 N. State St., Ste.3300, Los Angeles, CA 90033. email: echristi@usc.edu. 